\title{
Puzzling asteroid 21 Lutetia: our knowledge prior to the Rosetta fly-by ${ }^{\star}$
}

\author{
I. N. Belskaya ${ }^{1,2}$, S. Fornasier ${ }^{1,3}$, Yu. N. Krugly ${ }^{2}$, V. G. Shevchenko ${ }^{2}$, N. M. Gaftonyuk ${ }^{4}$, M. A. Barucci ${ }^{1}$, \\ M. Fulchignoni ${ }^{1,3}$, and R. Gil-Hutton ${ }^{5}$
}

1 LESIA, Observatoire de Paris, France e-mail: irina.belskaya@obspm.fr

2 Institute of Astronomy of Kharkiv Karazin National University, Kharkiv, Ukraine

3 Université de Paris 7 Denis Diderot, France

${ }^{4}$ Crimean Astrophysical Observatory, Crimea, Simeiz, Ukraine

5 Complejo Astronómico El Leoncito (Casleo) and Universidad Nacional de San Juan, San Juan, Argentina

Received 5 January 2010 / Accepted 2 March 2010

\section{ABSTRACT}

\begin{abstract}
Aims. A wide observational campaign was carried out in 2004-2009 that aimed to complete the ground-based investigation of Lutetia prior to the Rosetta fly-by in July 2010.

Methods. We obtained BVRI photometric and $V$-band polarimetric measurements over a wide range of phase angles, and visible and infrared spectra in the $0.4-2.4 \mu \mathrm{m}$ range. We analyze them with previously published data to retrieve information about Lutetia's surface properties.

Results. Values of lightcurve amplitudes, absolute magnitude, opposition effect, phase coefficient, and BVRI colors of Lutetia surface seen at near pole-on aspect are determined. We define more precisely parameters of polarization phase curve and show their distinct deviation from any other moderate-albedo asteroid. An indication of possible variations in both polarization and spectral data across the asteroid surface are found. To explain features found by different techniques, we propose that (i) Lutetia has a non-convex shape, probably due to a large crater, and heterogeneous surface properties probably related to surface morphology; (ii) at least part of the surface is covered by a fine-grained regolith of particle size smaller than $20 \mu \mathrm{m}$; (iii) the closest meteorite analogues of Lutetia's surface composition are particular types of carbonaceous chondrites, or Lutetia has specific surface composition that is not representative among studied meteorites.
\end{abstract}

Key words. minor planets, asteroids: individual: 21 Lutetia - techniques: photometric - techniques: spectroscopic techniques: polarimetric

\section{Introduction}

Asteroid 21 Lutetia has been extensively observed using different techniques for more than 30 years. The interest in this object was initially related to its classification as an M-type asteroid with a possible metallic composition (see Bowell et al. 1978). Since 2004 when Lutetia was selected as a target of the Rosetta mission, the volume of observational data for this asteroid has rapidly grown (see Barucci \& Fulchignoni 2009, for a review).

On the basis of photometric data obtained in 1962-1998, Torppa et al. (2003) determined the pole coordinates $\lambda_{\mathrm{p}}=$ $39^{\circ}\left(220^{\circ}\right), \beta_{\mathrm{p}}=3^{\circ}$ and the sidereal rotation period $P_{\text {sid }}=$ $8.165455 \mathrm{~h}$. The shape was found to have some irregular features with rough global dimensions $a / b=1.2$ and $b / c=1.2$. Drummond et al. (2009) provided new estimates of these parameters using adaptive optics images of Lutetia at the Keck telescope, of $\lambda_{\mathrm{p}}=49^{\circ}, \beta_{\mathrm{p}}=-8^{\circ}$ and a shape of $132 \times 101 \times 76 \mathrm{~km}$

* Based on observations carried out at the ESO-NTT (La Silla, Chile), the Telescopio Nazionale Galileo (La Palma, Spain), the Crimean Astrophysical Observatory (Ukraine), the Asiago Astrophysical Observatory (Italy) and Complejo Astronómico El Leoncito (Casleo, Argentine). with formal uncertainties of $1 \mathrm{~km}$ in the equatorial dimensions, and $31 \mathrm{~km}$ for the shortest axis.

On the basis of spectral and polarimetric observations, three types of meteorites are generally taken into consideration as possible analogues: iron meteorites (Bowell et al. 1978; Dollfus et al. 1979), enstatite chondrites (Chapman et al. 1975; Vernazza et al. 2009), and some types of carbonaceous chondrites, mainly CO3 or CV3 (Belskaya \& Lagerkvist 1996; Birlan et al. 2004; Barucci et al. 2008; Lazzarin et al. 2009). The main problem in spectral data interpretation is the featureless spectrum of Lutetia. A few minor features in the visible range were reported and interpreted as being indicative of aqueous alteration material consistent with carbonaceous chondrites composition (see Lazzarin et al. 2009, and references therein). A $3 \mu \mathrm{m}$ feature associated with hydrated minerals was found by Rivkin et al. (2000). In the emissivity spectra, a narrow $10 \mu \mathrm{m}$ emission feature was found (Feierberg et al. 1983; Barucci et al. 2008). It was interpreted as being indicative of fine silicate dust (Feierberg et al. 1983). According to Barucci et al. (2008), the emissivity spectrum is similar to that of the CO3 and CV3 carbonaceous chondrites with a grain size smaller than $20 \mu \mathrm{m}$.

To constrain the surface composition it is important to know Lutetia's albedo. However, the diversity in albedo estimates 
Table 1. Aspect data of photometric observations and magnitudes.

\begin{tabular}{|c|c|c|c|c|c|c|c|}
\hline $\begin{array}{l}\text { Date } \\
\text { (UT) }\end{array}$ & $\begin{array}{c}r \\
(\mathrm{AU})\end{array}$ & $\begin{array}{c}\Delta \\
(\mathrm{AU})\end{array}$ & $\begin{array}{c}\lambda \\
(\mathrm{deg})\end{array}$ & $\begin{array}{r}\beta \\
(\operatorname{deg})\end{array}$ & $\begin{array}{c}\alpha \\
(\mathrm{deg})\end{array}$ & $\begin{array}{c}V_{0}(1, \alpha) \\
(\mathrm{mag})\end{array}$ & Filter \\
\hline 20040916.07 & 2.163 & 1.416 & 47.72 & -3.86 & 22.17 & $8.32 \pm 0.03$ & $B V R I$ \\
\hline 20040917.06 & 2.164 & 1.408 & 47.74 & -3.86 & 21.87 & $8.35 \pm 0.04$ & $B V R I$ \\
\hline 20041007.06 & 2.195 & 1.285 & 46.31 & -3.93 & 14.12 & $8.06 \pm 0.03$ & $B V R I$ \\
\hline 20041008.09 & 2.197 & 1.281 & 46.15 & -3.93 & 13.65 & $8.04 \pm 0.02$ & $B V R I$ \\
\hline 20041110.81 & 2.254 & 1.274 & 38.40 & -3.34 & 4.75 & $7.63 \pm 0.02$ & $V$ \\
\hline 20081128.96 & 2.420 & 1.434 & 69.00 & -1.15 & 0.91 & - & V \\
\hline 20081129.76 & 2.421 & 1.435 & 68.79 & -1.12 & 0.58 & $7.28 \pm 0.02$ & $V$ \\
\hline 20081130.91 & 2.423 & 1.437 & 68.50 & -1.10 & 0.51 & $7.24 \pm 0.02$ & $V$ \\
\hline 20081201.96 & 2.425 & 1.440 & 68.22 & -1.08 & 0.89 & $7.30 \pm 0.02$ & $V$ \\
\hline 20081202.99 & 2.427 & 1.442 & 67.95 & -1.05 & 1.39 & $7.36 \pm 0.02$ & $V$ \\
\hline 20081203.86 & 2.429 & 1.445 & 67.74 & -1.03 & 1.81 & $7.40 \pm 0.02$ & $V$ \\
\hline 20081215.71 & 2.450 & 1.499 & 64.80 & -0.74 & 7.59 & $7.78 \pm 0.02$ & $V$ \\
\hline 20090310.80 & 2.593 & 2.569 & 70.60 & 0.63 & 22.18 & $8.28 \pm 0.03$ & $V R$ \\
\hline 20090311.75 & 2.594 & 2.583 & 70.87 & 0.64 & 22.12 & $8.25 \pm 0.03$ & $V R$ \\
\hline
\end{tabular}

by different techniques has been quite large, from 0.1 (Zellner et al. 1976) to 0.22 (Tedesco et al. 2002). Polarimetric means of albedo determination have provided contradictory results (Zellner et al. 1976; Gil-Hutton 2007). The accuracy of radiometric albedo measured strongly depends on the adopted absolute magnitude, which is not well-determined for Lutetia due to a lack of observations at small phase angles.

In this paper, we present new photometric, polarimetric and spectral observations of Lutetia carried out in 2004-2009. These observations were performed to determine absolute magnitude and albedo, and to place additional constraints on surface properties. We present an analysis of these data performed together with previously published data. This should be important not only for deriving physical characteristics of this particular asteroid but first of all for checking the efficiency of remote techniques in the study of atmosphereless bodies.

\section{Observations and results}

\subsection{Photometry}

The observations were carried out in 2004 using the $0.7-\mathrm{m}$ telescope of Chuguev Observational Station situated $70 \mathrm{~km}$ from Kharkiv, and in 2008-2009 using the 1-m telescope of the Crimean Astrophysical Observatory in Simeiz, Crimea. The 0.7-m telescope was equipped with a SBIG ST-6 UV camera mounted at the Newtonian focus $(f / 4)$. In Simeiz, we used a SBIG ST-6 camera placed on the 1-m Ritchey-Chretien telescope equipped with a focal reducer $(f / 5$ system $)$. The photometric reduction of the CCD frames was performed using the ASTPHOT package developed at DLR by Mottola (Mottola et al. 1994). The absolute calibration was performed using standard stars of colors close to those of the Sun taken from Landolt (1983, 1992) and Lasker et al. (1988). The measurements were obtained in the standard Johnson-Cousins photometric system. The method of CCD observations and data processing included all standard procedures and was described in detail by Krugly et al. (2002). The mean time of observations in UT, the heliocentric $(r)$ and geocentric $(\Delta)$ distances, the solar phase angle $(\alpha)$, the ecliptic longitude $(\lambda)$ and latitude $(\beta)$ in epoch J2000.0, the magnitude $V_{0}(1, \alpha)$ reduced to the lightcurve primary maximum and its estimated error, and finally photometric bands of observations are given in Table 1 . The estimated error in the absolute photometry includes both the uncertainty in photometric

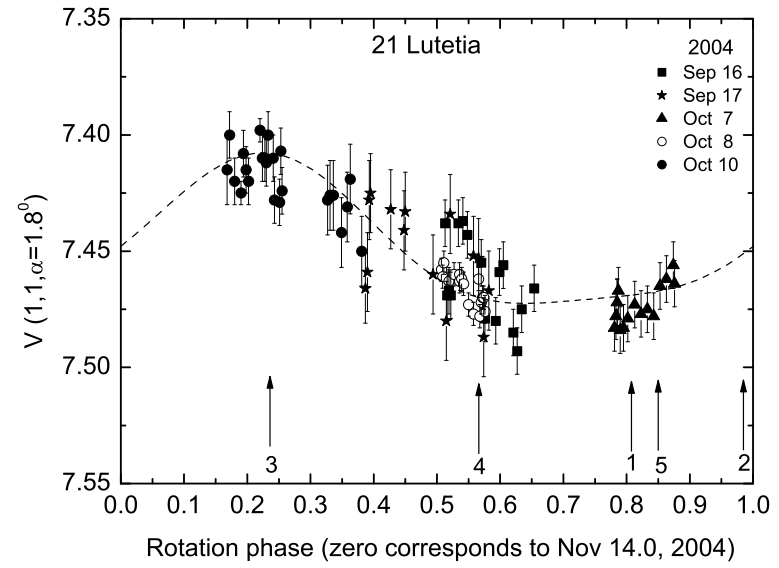

Fig. 1. Composite lightcurves of 21 Lutetia in 2004 apparition fitted with the Fourier fit. The arrows indicate rotation phases of our spectral observations (see Table 3 and Fig. 8).

reduction, typically $0.01-0.02^{\mathrm{m}}$, and the uncertainty in the lightcurve amplitude correction.

During our observations in 2004 we were unable to cover small phase angles due to bad weather conditions, and the observational program was continued in 2008. According to the latest estimates of Lutetia's pole coordinates $\lambda_{\mathrm{p}}=51^{\circ}\left(220^{\circ}\right)$, $\beta_{\mathrm{p}}=-4^{\circ}$ (B. Carry, personal communication), all of our observations were made close to the pole-on direction with an aspect angle $\approx 10^{\circ}$ in 2004 and $\approx 20^{\circ}$ in $2008-2009$. The composite lightcurves of each apparation are shown in Figs. 1 and 2. The lightcurve amplitude increased from $0.06^{\mathrm{m}}$ in 2004 , to $0.09^{\mathrm{m}}$ in 2008 , and $0.12^{\mathrm{m}}$ in 2009 at a phase angle as large as $22^{\circ}$. The lightcurves exibit an irregular behaviour with one pair of extrema. The measured lightcurve amplitudes and features are consistent with the observations of 1981 (Lupishko et al. 1983; Zappala et al. 1984) and 1985 (Lupishko et al. 1987; Dotto et al. 1992), which were also near pole-on aspect.

To obtain the phase function, we normalized all the data to the lightcurve primary maximum. Errors due to amplitude corrections were taken into account in the magnitude's uncertainties. We also used the $V$-magnitudes measured in 2004 at the phase angle of $27.4^{\circ}$ by Mueller et al. (2006) and normalized it to the lightcurve maximum using our lightcurve for the 2004 opposition. We applied the same procedure to the available observations of Lutetia from the 1981, 1983, and 1985 


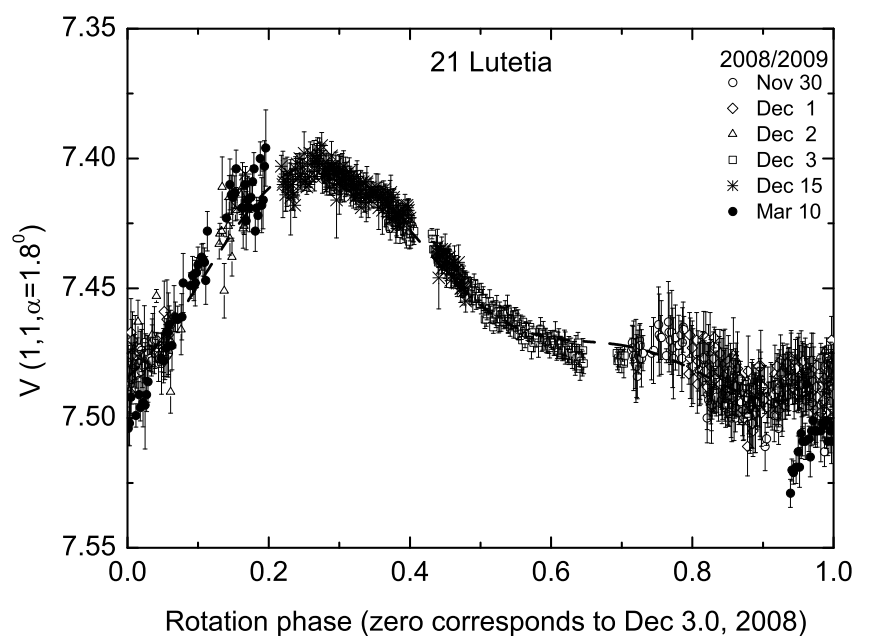

Fig. 2. Composite lightcurves of 21 Lutetia in 2008/2009 apparition fitted with the Fourier fit.

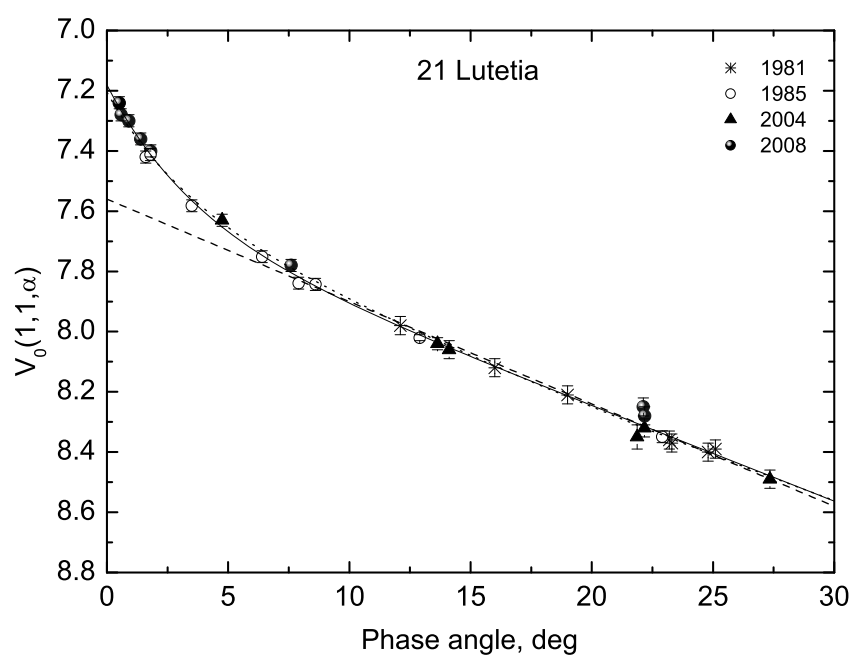

Fig. 3. Magnitude phase dependence for Lutetia based on observations at different apparitions at near polar aspects fitted by the HG function (the dotted line) and the linear-exponential function (the solid line). The dashed line shows liner fit to the data at $\alpha \geq 7^{\circ}$.

oppositions, separately for each opposition. These data were obtained by different authors (Lupishko et al. 1983, 1987; Dotto et al. 1992; Lagerkvist et al. 1995; Zappala et al. 1984) at a variety of phase angles and were not analyzed jointly. In our analysis, we used an updated value of Lutetia's sidereal rotation period $P_{\text {sid }}=8.168268 \mathrm{~h}$ and normalized all the data to the same maximum.

We found that observations in the four oppositions corresponding to pole-on aspect are mutually in good agreement within the error bars. The phase function obtained is shown in Fig. 3. Fitting the data with both a HG fit (Bowell et al. 1989) and with a linear-exponential fit (Kaasalainen et al. 2003), we obtained practically identical curves. The HG-fit to the phase curve normalized to the lightcurve primary maximum inferred $H=7.20 \pm 0.01$ and $G=0.12 \pm 0.01$. We note that, for the phase function normalized to the mean lightcurve, $H=7.25 \pm 0.01$. The phase coefficient obtained by the linear fit to the data at phase angles $\alpha \geq 7^{\circ}$ is equal to $\beta=0.034 \pm 0.001 \mathrm{~m} / \mathrm{deg}$ and the magnitude at zero phase angle corresponding to the extrapolation of the linear fit is $V(1,0)=7.56 \pm 0.01$. The amplitude of the opposition effect, defined as an increase in magnitude above the linear fit at zero phase angle, was estimated to be $0.36^{\mathrm{m}}$. Both the opposition effect amplitude and the value of the phase slope are consistent with a moderate-albedo surface. Based on the empirical correlation between phase coefficient and albedo (Belskaya \& Shevchenko 2000), an average albedo in the range of 0.12-0.20 is expected for Lutetia's surface.

The phase function obtained for the observations in 1983 at near-equatorial aspects is characterized by systematically lower magnitudes that are described well by the $H G$-function with $H=$ $7.29 \pm 0.02$ and $G=0.13 \pm 0.03$. Thus, the difference between the absolute magnitudes at near polar and near equatorial aspects is found to be as small as $0.1^{\mathrm{m}}$. It implies that an upper limit to Lutetia's shape elongation is given by $b / c \leq 1.1$ in the case of a homogeneous surface albedo.

We also measured the BVRI colors of Lutetia at different phase angles and found a slight increasing trend toward larger phase angles, not exceeding a level of $0.001^{\mathrm{m}} / \mathrm{deg}$. The mean measured colors are $B-V=0.65 \pm 0.01, V-R=0.42 \pm 0.01$, and $V-I=0.76 \pm 0.01$.

\subsection{Polarimetry}

The first polarimetric observations of Lutetia were performed in 1973 by Zellner \& Gradie (1976). They derived a polarimetric slope $h=0.169 \% / \mathrm{deg}$ in the green filter with an effective wavelength of $0.52 \mu \mathrm{m}$ and an albedo of 0.10 based on the empirical relationship " $h$-albedo". They also measured the inversion angle $\alpha_{\text {inv }}=24.2^{\circ}$, which appeared to be the largest of all asteroids in their data-set. Polarimetric observations of Lutetia were successively carried out in 1985 in $U B V R I$ filters at a phase angle of 7.5 deg close to the polarization minimum (Belskaya et al. 1987). These data revealed that the depth of polarimetric minimum reached $1.3 \%$ in the $V$ band and slightly increased with wavelength. Other observations of Lutetia were carried out in the framework of a coordinate program at three observatories: the Crimean Astrophysical Observatory (Ukraine), the Asiago Observatory (Italy) and Complejo Astronómico El Leoncito (Casleo, Argentine), to cover phase angles that had not been previously observed. A part of these data was published among results of observations at each telescope (Fornasier et al. 2006; Gil-Hutton 2007; Belskaya et al. 2009). Here we report complementary observations of Lutetia that have not yet been published. Table 2 presents the mean time of observations in UT, the phase angle $\alpha$, the polarization degree $P$ and position angle $\Theta$ in the equatorial coordinate system, together with the root-mean-square errors $\sigma_{P}$ and $\sigma_{\Theta}$, the calculated values of the corresponding $P_{r}$ and position angle $\Theta_{r}$ in the coordinate system referring to the scattering plane as defined by Zellner \& Gradie (1976), and the telescope. Methods of observations and data processing were identical to those described by Fornasier et al. (2006) for Asiago, Belskaya et al. (2009) for Crimea, and Gil-Hutton (2007) for Casleo. The polarization-phase function of Lutetia obtained using both new and published data is shown in Fig. 4. The data were fitted with a linear-exponential function as described by Kaasalainen et al. (2003). Similar curves were obtained by fitting the data with either a trigonometric fit (Lumme \& Muinonen 1993) or parabolic fit. The scatter of the data, which is rather large and exceeds the estimated errors in each measurement, may be indicative of a variation in polarization degree across the asteroid surface.

We analyzed the deviations of the polarization degree from the fitted phase curve and found that they are of a systematic rather than a random nature. Figure 5 plots these deviations versus rotation phase for observations in 1973 corresponding to the 
Table 2. Results of polarimetric V-band observations of 21 Lutetia.

\begin{tabular}{crccccccc}
\hline \hline $\begin{array}{c}\text { Date } \\
\text { UT }\end{array}$ & $\begin{array}{r}\alpha \\
(\mathrm{deg})\end{array}$ & $\begin{array}{c}P \\
(\%)\end{array}$ & $\begin{array}{c}\sigma_{P} \\
(\%)\end{array}$ & $\begin{array}{c}\Theta \\
(\mathrm{deg})\end{array}$ & $\begin{array}{c}\sigma_{\Theta} \\
(\mathrm{deg})\end{array}$ & $\begin{array}{c}P_{r} \\
(\%)\end{array}$ & $\begin{array}{c}\Theta_{r} \\
(\mathrm{deg})\end{array}$ & Tel. \\
\hline 20041017.96 & 8.75 & 1.37 & 0.09 & 80.8 & 1.9 & -1.36 & 87.0 & 1 \\
20060406.79 & 17.38 & 0.81 & 0.07 & 97.0 & 2.0 & -0.72 & 78.1 & 2 \\
20081031.30 & 14.80 & 1.28 & 0.02 & 93.0 & 0.5 & -1.25 & 97.0 & 3 \\
20081104.25 & 13.10 & 1.31 & 0.02 & 90.9 & 0.4 & -1.29 & 94.8 & 3 \\
\hline
\end{tabular}

Notes. 1. 1.25 m, Crimea. 2. 1.82 m, Asiago. 3. 2.15 m, Casleo.

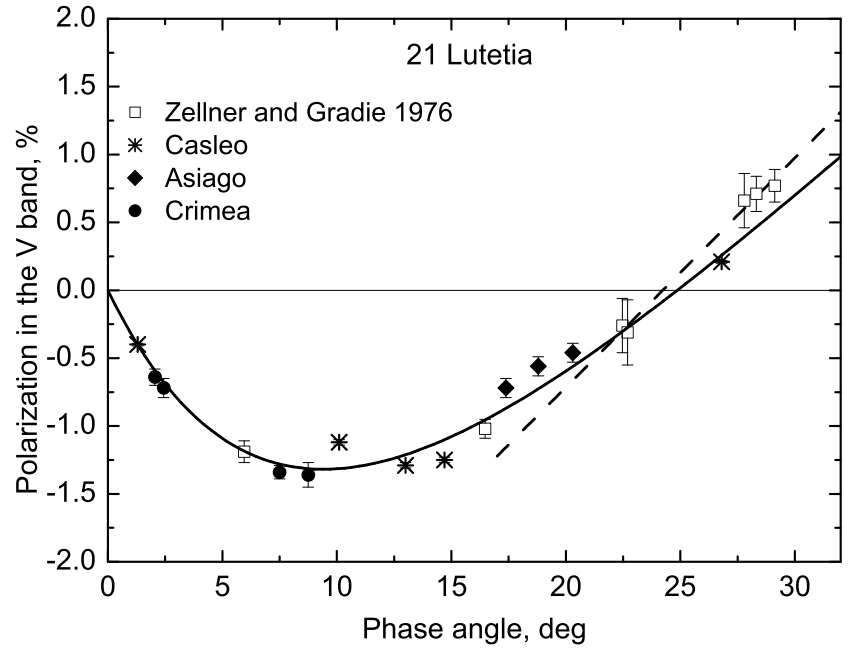

Fig. 4. Polarization phase dependence for Lutetia based on observations in 1973-2008 at different observational sites fitted by the linearexponential function (solid line). The dashed line indicates the polarimetric slope h as defined by Zellner \& Gradie (1976).

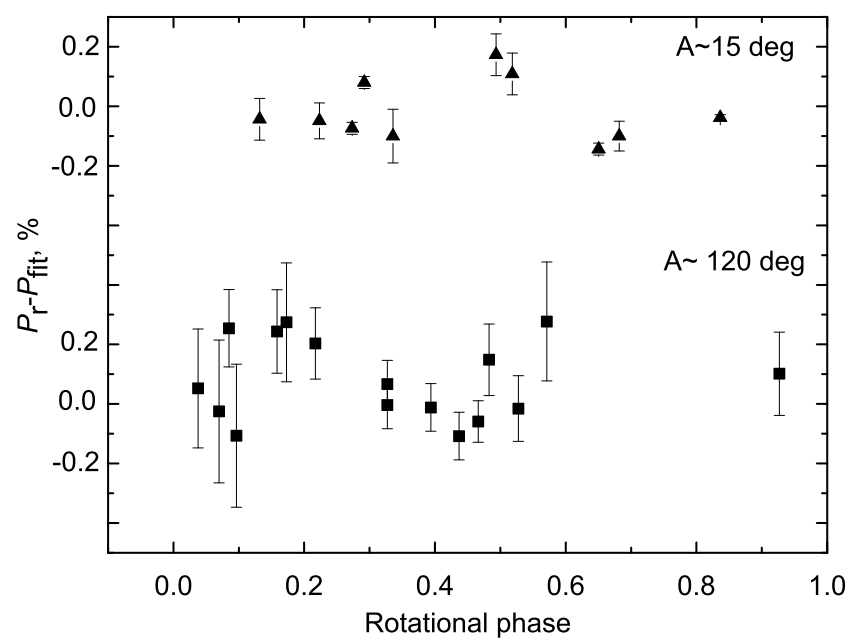

Fig. 5. The deviations of the polarization degree $\left(P_{r}\right)$ from the linearexponential fit to polarization phase curve $\left(P_{\text {fit }}\right)$ versus rotation phase at different aspect angle $A$.

aspect angle of about $120^{\circ}$, and in 2004 and 2008 oppositions when the aspect was close to pole-on $\left(6-24^{\circ}\right)$. One can see that variations in the polarization degree tend to increase toward equatorial aspect and can reach up to $0.2 \%$.

The amplitude of variations in polarization degree across the Lutetia's surface resembles that measured on asteroid 4 Vesta (e.g., Lupishko et al. 1988) and could have the same cause, i.e. macroscale surface heterogeneity. The mean polarization phase

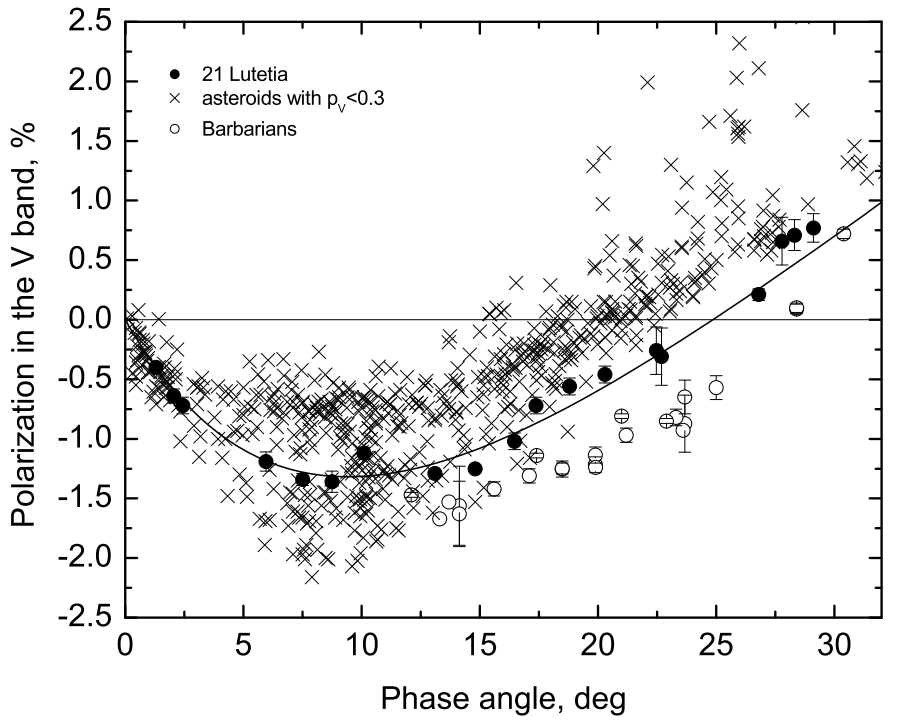

Fig. 6. The polarization-phase dependence of Lutetia (solid line) compared with available observations of moderate and low albedo asteroids (crosses) taken from the Asteroid Polarimetric Database (Lupishko \& Vasilyev 2008) and data for Barbara-like asteroids (circles) from Cellino et al. (2006), Gil-Hutton et al. (2008), and Masiero \& Cellino (2009).

dependence of Lutetia is characterized by the parameters $P_{\min }=$ $-1.30 \pm 0.07 \%, \alpha_{\min }=9.1 \pm 0.8 \mathrm{deg}, \alpha_{\mathrm{inv}}=25.0 \pm 0.4 \mathrm{deg}$, and $h=0.131 \pm 0.009 \% / \mathrm{deg}$.

The polarimetric slope $h$ has a smaller value than that defined by Zellner \& Gradie (1976). It corresponds to the geometric albedo $p_{V}=0.13 \pm 0.02$ when using the empirical relationship " $h$-albedo" based on meteorite data (Zellner \& Gradie 1976), and $p_{V}=0.16 \pm 0.02$ using the calibration based on IRAS albedos (Cellino et al. 1999). The difference between these two values is caused by the different calibration scales of albedos. Albedos of meteorites were measured in the laboratory at phase angle $\alpha=5^{\circ}$ while IRAS albedos were determined at zero phase angle using asteroid absolute magnitude $H$.

The most interesting polarimetric characteristic of Lutetia is its wide branch of negative polarization. Figure 6 compares Lutetia's data with available polarimetric measurements of low and moderate albedo asteroids. In this comparison, we used the Asteroid Polarimetric Database (Lupishko \& Vasilyev 2008) selecting data of an accuracy superior to $0.2 \%$ for asteroids of albedo less than 0.3 . Lutetia's observations characterized by an inversion angle as large as $25^{\circ}$ represent a marginal case compared to a variety of asteroids observed so far. Only asteroid 234 Barbara and four other asteroids called "Barbarians" exhibited a polarization branch wider than that of Lutetia (see Fig. 6). This group of moderate-albedo asteroids of spectral type L, K 
or Ld have anomalous polarization properties which may be related to their specific surface composition (Cellino et al. 2006; Gil-Hutton et al. 2008; Masiero \& Cellino 2009). We note, that the polarization minimum value of these asteroids deviates considerably from the well-known correlation " $P_{\min }$-albedo" and cannot be used for albedo estimation. In the case of Lutetia, this correlation also fails.

A negative polarization can be explained by several physical mechanisms, the most appropriate of which appear to be the coherent backscattering mechanism (see Shkuratov et al. 1994, for a review) and the single particle scattering (e.g., Muñoz et al. 2000). The coherent backscattering mechanism contributes to both the brightness opposition effect and the negative polarization branch and is particularly efficient for high albedo surfaces producing narrow backscattering peaks (Mishchenko et al. 2006). The measured phase curves of Lutetia (Figs. 3 and 4) do not exibit any sharp features toward zero phase angle. Both phase curves are characterized by wide opposition effects that assume relatively small contribution from the coherent backscattering. The contribution of the mechanism of the single particle scattering remains poorly understood but its efficiency in producing wide negative polarization branch has been demonstrated by laboratory and numerical modeling (e.g. Muñoz et al. 2000; Shkuratov et al. 2002). The negative branch was found to become more prominent and the inversion angle to increase in the cases of a) an increase in the refractive index; b) a decrease in the particle sizes to sizes comparable wavelength; c) complex internal structure of particles; and d) the mixture of particles with high contrast in albedo (Muñoz et al. 2000; Shkuratov et al. 1994, 2002; Zubko et al. 2005). One or several of the abovementioned properties can be responsible for the particular polarization characteristics of Lutetia.

On the basis of the relationship between $P_{\min }$ and $\alpha_{\text {inv }}$, Dollfus et al. (1975) noted that 21 Lutetia belongs to a group with a regolith of fines. This group was separated on the basis of the measurements of lunar fines with average grain sizes of the order of $10 \mu \mathrm{m}$ across a range from smaller than $1 \mu \mathrm{m}$ to several tens of microns (e.g., Geake \& Dollfus 1986). Lutetia's data were later interpreted as being indicative of a metallic surface with a grain size of $20-40 \mu \mathrm{m}$ (Dollfus et al. 1979). This conclusion was based on measurements of specific powders, such as titanium, dural, limonite, carbonyl iron globules, while the properties of neither pulverized iron meteorites nor pulverized enstatite chondrites were consistent with the polarimetric curves of M-type asteroids. Laboratory measurements of iron meteorites and enstatite chondrites with particle sizes smaller than $50 \mu \mathrm{m}$ infer smaller inversion angles than measured for Lutetia's surface (Lupishko \& Belskaya 1989). A CV3 type of carbonaceous chondrites was mentioned as the closest polarimetric analogue of Lutetia (Belskaya \& Lagerkvist 1996).

Figure 7 shows an updated relationship between $P_{\min }$ and $\alpha_{\text {inv }}$ for asteroids and meteorites. Among meteorites, the widest negative polarization branches are found for $\mathrm{CV} 3$ and $\mathrm{CO} 3$ types of carbonaceous chondrites. These types of chondrites are distinguished by their relative abundances of refractory inclusions, in particular calcium-aluminum rich inclusions (CAI) (e.g. Scott \& Krot 2005). Sunshine et al. (2008) assumed that the presence in some CAIs of spinel, which has one of the highest indices of refraction among meteorite minerals, may explain the large inversion angles. Another possible explanation is related to the fine structure of $\mathrm{CV} 3$ and $\mathrm{CO} 3$ meteorite samples measured with the polarimetric technique. The measurements of cleavage faces of solid pieces and pulverized samples for CV3 Allende and CO3 Kanzas chondrites (Shkuratov et al. 1984) showed that the depth

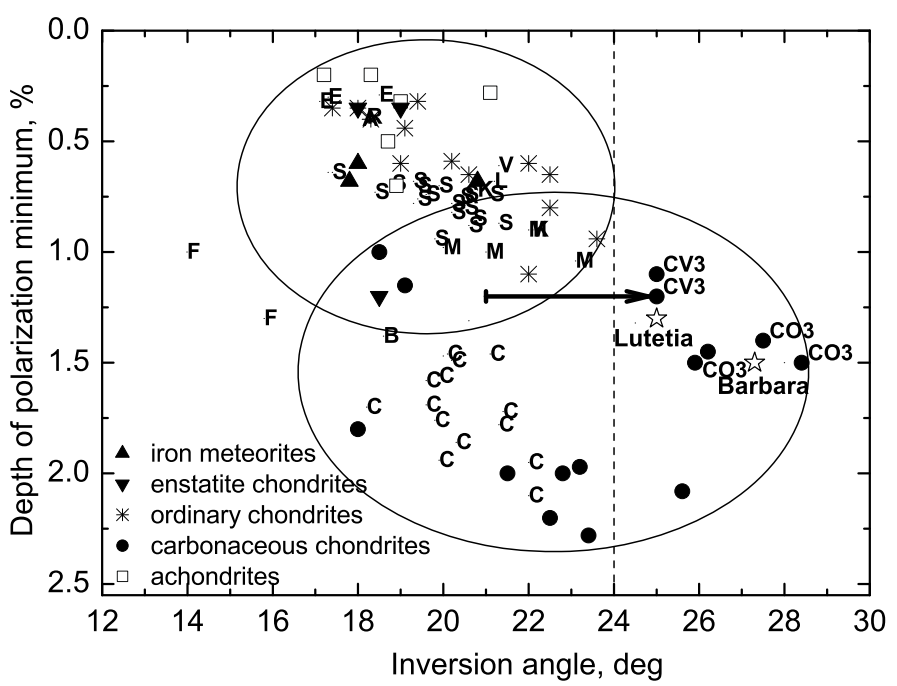

Fig. 7. Minimum polarization $P_{\text {min }}$ (in absolute term) versus inversion angle for asteroids and meteorites. The two ellipses outline the location of carbonaceous chondrites and all other types of meteorites. The arrow shows the changes in the inversion angle for the CV3 chondrite Allende when these angles are measured for either a solid piece or a crushed sample. Data for meteorites are taken from Zellner et al. (1977), Geake \& Dollfus (1986), Shkuratov et al. (1984), Lupishko \& Belskaya (1989). Asteroid polarimetric parameters were calculated by fitting a linear-exponentional function to the data for individual asteroids in the Asteroid Polarimetric Database. Letters designate taxonomic class of asteroids according to the classification scheme of Tholen (1989).

of the negative branch was practically identical for powder and solid samples while the inversion angle noticeably increased for a powder sample (see Fig. 7). However it is difficult to explain why pulverized samples of other types of carbonaceous chondrites have smaller inversion angles.

Laboratory measurements are presently available for a rather limited sample of meteorites that do not cover all known meteorite classes. None of the measured iron meteorites nor enstatite and ordinary chondrites exhibited an inversion angle as large as found for Lutetia. Only particular types of carbonaceous chondrites are found to have a wide negative polarization branch. It is possible that a fine-grained mixture of components with highly different optical properties (carbon, silicates, irons) is required to produce the large inversion angle seen for Lutetia.

\subsection{Spectral observations}

The observations were performed during two runs in November 2004 at the TNG telescope at la Palma, Spain and in January 2007 at the NTT telescope of the European Southern Observatory in Chile.

At the TNG telescope, we used the DOLORES spectrometer with two grisms: the low resolution red grism (LR-R) covering the $0.51-0.95 \mu \mathrm{m}$ range with a spectral dispersion of $2.9 \AA / \mathrm{px}$ and the medium resolution blue grism MR-B covering the $0.4-$ $0.7 \mu \mathrm{m}$ range with a dispersion of $1.7 \AA / \mathrm{px}$. The spectra obtained were separately reduced and then combined together to obtain a spectral coverage from 0.4 to $0.95 \mu \mathrm{m}$. For the infrared range, we used the near infrared camera and spectrometer (NICS) equipped with an Amici prism disperser covering the 0.85-2.4 $\mu \mathrm{m}$ range.

At the NTT telescope, visible spectra were acquired using the EMMI instrument with the grism covering the wavelength range of $0.41-0.96 \mu \mathrm{m}$ with a dispersion of $3.1 \AA / \mathrm{px}$. The data acquisition and reduction techniques are described by 
Table 3. Description of spectral observations of 21 Lutetia.

\begin{tabular}{cccccccc}
\hline \hline Date & $\begin{array}{c}\text { UT-start } \\
\text { (hh:mm) }\end{array}$ & $\begin{array}{c}T_{\text {exp }} \\
(\mathrm{s})\end{array}$ & Tel. & Instr. & Grism & Airm. & $\begin{array}{c}\text { Solar } \\
\text { analog }\end{array}$ \\
\hline 20041104 & $23: 35$ & 40 & TNG & DOLORES & LR-R & 1.05 & 1 \\
20041115 & $23: 37$ & 40 & TNG & DOLORES & MR-B & 1.05 & 1 \\
20041116 & $01: 10$ & 40 & TNG & DOLORES & LR-R & 1.11 & 1 \\
20041116 & $01: 12$ & 40 & TNG & DOLORES & MR-B & 1.11 & 1 \\
20041116 & $03: 06$ & 40 & TNG & DOLORES & LR-R & 1.50 & 1 \\
20041116 & $03: 08$ & 40 & TNG & DOLORES & MR-B & 1.51 & 1 \\
20041118 & $23: 11$ & 60 & TNG & NICS & AMICI & 1.05 & 1 \\
20070120 & $08: 47$ & 120 & NTT & EMMI & GR1 & 1.55 & 2 \\
20070120 & $08: 42$ & 240 & NTT & EMMI & GR5 & 1.58 & 2 \\
\hline
\end{tabular}

Notes. 1. Hyades64 (airmass 1.03). 2. La102-1081 (airmass 1.22).

Fornasier et al. (2008). The observational details are summarized in Table 3, which contains the date and UT-time at the start of observations, the exposure time, telescope, instrument, airmass of the object, the name and the airmass of solar analog star, and the number corresponding to the rotation phase at the time of the observation, as shown in Fig. 1.

The spectral data are presented in Fig. 8. Three visible spectra measured on Nov. 15/16 $\left(\alpha=7.3^{\circ}\right)$ at different rotation phases (see arrows in Fig. 1) show noticeably different shapes. In two spectra (1 and 2), a broad band at $0.45-0.55 \mu \mathrm{m}$ is clearly visible, while in the spectrum close to the lightcurve maximum (3) it is less evident. For comparison, we also presented the spectrum taken on May 26, 2004 by Barucci et al. (2005) at close to the same pole-on aspect but at a larger phase angle $\left(\alpha=24^{\circ}\right)$, which does not contain a broad band at $0.45-0.55 \mu \mathrm{m}$. This band is also not seen in the spectrum taken in 2007. The spectrum corresponds to the opposite side of Lutetia from that covered by the spectra taken in 2004. The faint absorption around $0.83 \mu \mathrm{m}$ seen in the spectrum is probably caused by the incomplete removal of telluric bands. On the other hand, the faint absorption feature around $0.43 \mu \mathrm{m}$ appears in all our spectra and appears to be real.

The near-infrared spectrum measured on Nov. 18, 2004 at the phase angle of $\alpha=8.8^{\circ}$ is flat with a small negative slope. It does not show any features detectable within the noise of the data. We compared it with the spectrum obtained by Birlan et al. (2006) at the same opposition but at a larger phase angle $\alpha=28.3^{\circ}$ and did not detect any phase angle effect. Both spectra acquired at almost the same rotational phase are flat. The reddening at relatively high phase angle is not seen for Lutetia's surface in the spectral range of $0.8-2.5 \mu \mathrm{m}$.

Our visible spectra are in a good agreement with previous observations of Lutetia. In some of our spectra, we confirmed the presence of a broad feature at $0.45-0.55 \mu \mathrm{m}$ previously reported by Lazzarin et al. (2009) for observations of the same 2004 apparition. Lazzarin et al. (2009) attributed this feature to a superposition of several absorption bands caused by a charge transfer involving various metal ions in pyroxenes. The faint absorption feature around $0.43 \mu \mathrm{m}$ was not detected in the 2004 spectra by Lazzarin et al. (2009) but was identified in some Lutetia spectra obtained in both 2000 (Busarev et al. 2004; Prokof'eva et al. 2005) and in 2003 (Lazzarin et al. 2004). The feature was interpreted to be indicative of aqueous alteration activity (Lazzarin et al. 2004, 2009; Prokof' eva et al. 2005).

Both the features and the overall shape of spectrum appear tend to change with Lutetia's rotation. Variations in spectral slope over the surface were found previously by Nedelcu et al. (2007) in the near-infrared wavelength range and by Busarev (2008) in the visible range. These data are related to
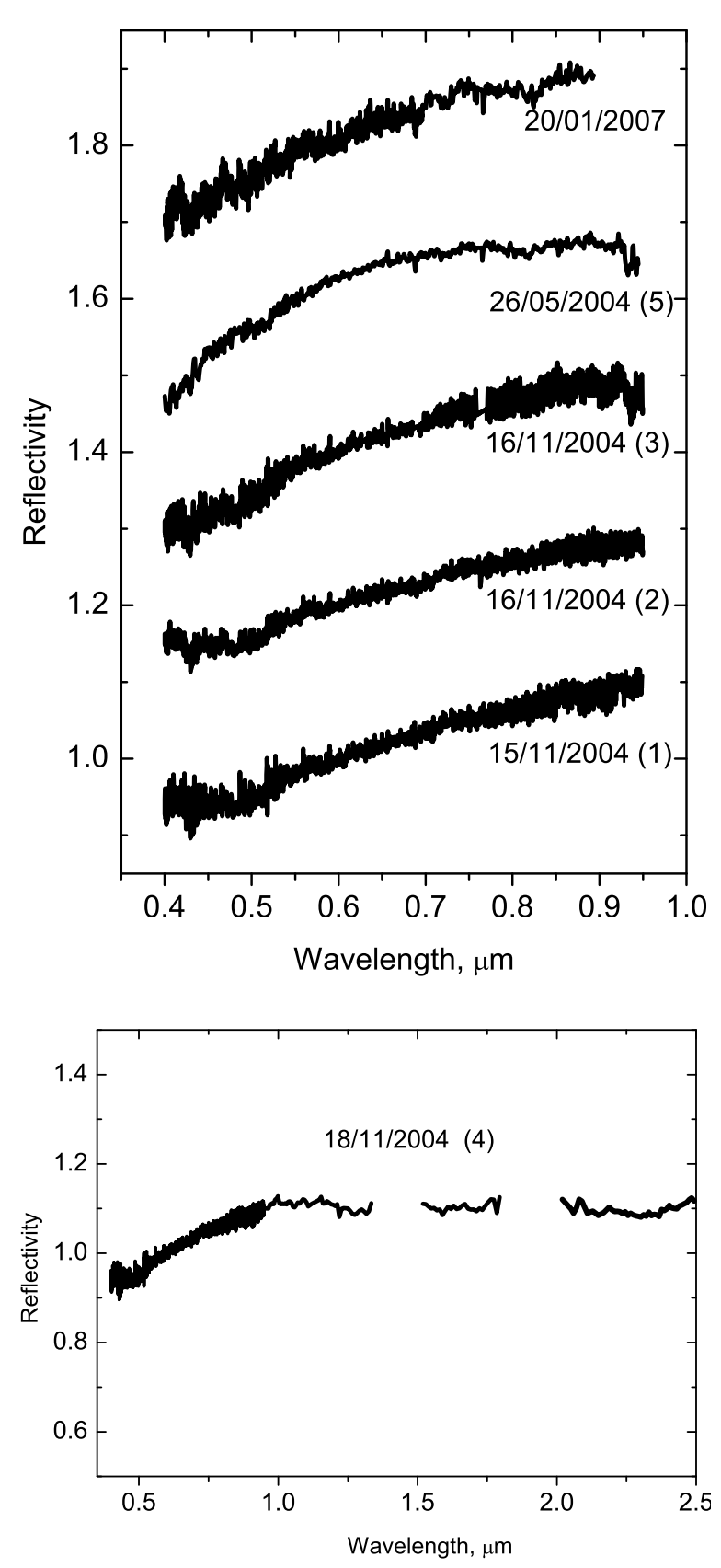

Fig. 8. Visual and near-infrared spectra of Lutetia. The spectra have been shifted by 0.2 for clarity. The numbers in parentheses correspond to the numbers in Fig. 1. The spectrum on May 26, 2004 was taken from Barucci et al. (2005). 
the equatorial aspect and were interpreted as being indicative of variations in the surface mineralogy (Nedelcu et al. 2007). Our data corresponding to the aspect angle of 14 deg appear to confirm surface heterogeneity for Lutetia.

\section{Discussion}

We have described the results of photometric, polarimetric and spectral observations of Lutetia that indicate the use of different techniques provides a rather consistent picture of the main physical and optical properties of the asteroid which appears to have a highly heterogeneous surface. The conclusion follows from: 1) the non-zero lightcurve amplitude measured at the polar aspect; 2) spectral slope variations found at both the near polar and equatorial aspects; and 3) observed variations in polarization degree over the surface. These features could be explained by assuming a global non-convex shape (e.g. caused by a large crater) and a heterogeneous surface texture and/or mineralogy. The hypothesis of a large crater in the northern hemisphere was also proposed by Carvano et al. (2008) to explain the value of Lutetia's albedo $p_{V}=0.13$ derived from their thermophysical model, which was smaller than the previous value of radiometric albedo $p_{V}=0.22$ obtained by Mueller et al. (2006).

We have no strong evidence in favor of large albedo variegations over Lutetia's surface. Available radiometric measurements performed for different aspects infer rather consistent values of Lutetia's albedo in the range of $0.19-0.22$ with an estimated uncertainty of 0.02 (Tedesco et al. 2002; Mueller et al. 2006; Lamy et al. 2008). Our new estimation of the polarization albedo of $0.16 \pm 0.02$ remains lower than radiometric albedo. However, it was shown in Sect. 2.2 that the determination of Lutetia's albedo from polarimetric data can be difficult because of the particular polarization properties of this asteroid. The measured values of opposition effect and phase slope are consistent with a moderate-albedo surface.

Using our precise determination of the absolute magnitude of Lutetia $H=7.25 \mathrm{mag}$ for the near polar aspect (corresponding to observations in 2004 and 2008), we calculated its albedo from available size estimates for these apparitions. The albedo ranges from 0.18 for an assumed effective diameter of $110 \mathrm{~km}$ (Drummond et al. 2009) to 0.22 for an effective diameter of $100 \mathrm{~km}$ (Mueller et al. 2006). The above values of albedo correspond to zero phase angle and cannot be directly compared to the albedos of meteorites, which are usually measured at $\alpha \approx 3-5^{\circ}$. We calculated the so-called four-degree albedo proposed by Shevchenko \& Tedesco (2006) using $V\left(1, \alpha=4^{\circ}\right)=7.63^{\mathrm{m}}$. This value roughly corresponds to the absolute magnitude of the asteroid without taking into account the opposition surge. The four-degree albedo of Lutetia is in the range of $0.13-0.16$ for an effective diameter in the range of $100-110 \mathrm{~km}$. These values of albedo are consistent with particular types of carbonaceous chondrites and enstatite chondrites and are smaller than typical values for iron meteorites (e.g. Gaffey 1976).

To compare the albedo and spectral properties of Lutetia with laboratory measurements, we need to take into account that these properties are sensitive to particle size. On the basis of the available data, we expect Lutetia's surface to be covered with fine-grained regolith. This conclusion follows from 1) the polarimetric properties of Lutetia characterizing by large inversion angle, and 2) the behaviour of the emissivity spectra of Lutetia with a narrow $10 \mu \mathrm{m}$ emission feature (Feierberg et al. 1983; Barucci et al. 2008). According to estimations, at least a portion of Lutetia's surface should be covered by fine regolith with a grain size $\leq 20 \mu \mathrm{m}$.
Fine-grained mixtures of components with different optical properties (irons, silicates, carbon) can drastically alter the spectral reflectivity and suppress silicate bands (e.g. Feierberg et al. 1982). The particle size is not well-controlled in laboratory measurements of crushed meteorites because of the different fragility of the meteorite components. Moreover, the processes that can affect the optical properties of regolith exposed to space are not enough understandable to confidently interpret asteroid spectra (see Chapman 2004, for review). It is possible that the observed variations in spectral properties of Lutetia are related to the different exposure history of its regolith due to a large impact.

Both spectral and polarimetric observations indicate that Lutetia's surface properties are quite different from those of most asteroids studied so far. In a new asteroid taxonomy, Lutetia was classified in the Xc subclass (DeMeo et al. 2009), to which very few members belong, among them 97 Klotho, which has spectral properties similar to those of Lutetia (Vernazza et al. 2009). The polarization properties of Klotho (Belskaya et al. 2009) also resemble those of Lutetia and deviate distinctly from those of other moderate-albedo asteroids. We expect that these two bodies have a very similar surface composition. According to Vernazza et al. (2009), they are most probable candidates to be the parent bodies of enstatite chondrites. This conclusion has difficulties in explaining 1) the observed features in the Lutetia's visible spectra which are interpreted as being indicative of aqueous alteration material (Lazzarin et al. 2004, 2009; Busarev 2004); 2) the presence of a $3 \mu \mathrm{m}$ feature associated with hydrated minerals (Rivkin et al. 2000); 3) the features of 5.2-38 $\mu \mathrm{m}$ emissivity spectrum (Barucci et al. 2008); and 4) the particular polarization properties of Lutetia. The above-mentioned features can be more naturally explained by assuming similarity of Lutetia's surface to particular types of carbonaceous chondrites. In turn, this assumption requires an explanation of relatively flat spectral slope of Lutetia toward ultraviolet wavelengths. Lazzarin et al. (2009) suggested several possible explanations but not exclude that available meteorite assemblages might not be representative of the Lutetia surface composition.

All of the above mentioned data are related to the surface properties of Lutetia. To constrain the interior composition, we need to estimate the mass and density of the asteroid. Although Prokof'eva-Mikhailovskaya et al. (2007) concluded about a complex satellite system of Lutetia, no satellites have yet been detected around the asteroid (Busch et al. 2009). The only available mass estimations of Lutetia come from the astrometric method and infer a density comparable to those of iron meteorites (Baer et al. 2009). However, available radar observations raise doubts about the reliability of the estimated mass. The radar albedo of Lutetia span from $0.17 \pm 0.07$ (Magri et al. 1999) to $0.24 \pm 0.07$ (Shepard et al. 2008) and both exclude a metallic surface composition. Radar data are consistent with the composition being similar to either enstantite chondrites or particular metal-rich $\mathrm{CH}$ type of carbonaceous chondrites (Shepard et al. 2010). A possible similarity with a CO/CV composition is also not excluded within the available uncertainties.

Observed variations in spectral and polarimetric properties across Lutetia's surface can be attributed not only to the heterogeneity of the surface texture but also of the surface composition, e.g. due to contamination during a large impact, which might also explain particular properties of Lutetia. However, neither satellites nor family members have yet been found for this asteroid. Previously classified as a member of Nysa family (Williams 1989), Lutetia does not belong to any family in later classifications (e.g., Zappala et al. 1995). Further study of these questions is needed. We note that the existence of satellites smaller 
than $6 \mathrm{~km}$ in diameter is not excluded by available observations (Busch et al. 2009).

\section{Conclusions}

On the basis of a detailed analysis of new photometric, polarimetric, and spectral data of the asteroid 21 Lutetia, together with observational data from the literature, we can draw some conclusions, which should be checked during the Rosetta fly-by:

- Lutetia has a non-convex shape, probably due to a large crater, and a heterogeneous surface properties, probably due to variations in the texture and/or mineralogy related to the surface morphology.

- At least part of Lutetia's surface is covered by regolith composed of particles with a mean grain size smaller than $20 \mu \mathrm{m}$.

- The closest meteorite analogues of Lutetia's surface composition are particular types of carbonaceous chondrites (CO, $\mathrm{CV}, \mathrm{CH}$ ). It is also possible that Lutetia has a specific surface composition that is not representative among studied meteorites or has a mixed mineralogy, e.g. due to surface contamination.

Flyby observations of Lutetia by the Rosetta spacecraft in July 2010 will provide a cross-check and verification of Earth-based remote sensing.

Acknowledgements. The research of INB has been supported by a Marie Curie Fellowship of the European Community. R.G.-H. acknowledges the support from CONICET through grant PIP114-200801-00205, and the partial financial support by CICITCA, Universidad Nacional de San Juan, through a research grant. We thanks to B. Carry for providing results on Lutetia's rotation properties prior to publication.

\section{References}

Baer, J., Chesley, S., \& Britt, D. 2009, Asteroid Masses V1.0. EAR-A-COMPIL5-ASTMASS-V1.0. NASA PDS

Barucci, M. A., \& Fulchignoni, M. 2009, in Rosetta: ESA's Mission to the Origin of the Solar System (Springer Science), 55

Barucci, M. A., Fulchignoni, M., Fornasier, S., et al. 2005, A\&A, 430, 313

Barucci, M. A., Fornasier, S., Dotto, E. et al. 2008, A\&A, 477, 665

Belskaya, I. N., \& Lagerkvist, C.-I. 1996, Planet. Space Sci., 44, 783

Belskaya, I. N., \& Shevchenko, V. G. 2000, Icarus, 147, 94

Belskaya, I. N., Lupishko, D. F., \& Shakhovskoj, N. M. 1987, Pis'ma Astron. Zh., 13, 530

Belskaya, I. N., Levasseur-Regourd, A.-C., Cellino, A., et al. 2009, Icarus, 199, 97

Birlan, M., Barucci, M. A., Vernazza, P., et al. 2004, New Astron., 9, 343

Birlan, M., Vernazza, P., Fulchignoni, M., et al. 2006, A\&A, 454, 677

Bowell, E., Chapman, C. R., Gradie, J. C., Morrison, D., \& Zellner, B. 1978, Icarus, 35, 313

Bowell, E., Hapke, B., Domingue, D., et al. 1989, in Asteroids II (Univ. of Arizona Press), 524

Busarev, V. V. 2008, In Asteroids, Comets, Meteors 2008, July 14-18, LPI Contr., 1405

Busarev, V. V., Bochkov, V. V., Prokof'eva, V. V., \& Taran, M. N. 2004, in The New Rosetta Targets (ASSL Kluwer), 79

Busch, M. W., Kulkarni, S. R., \& Conrad, A. 2009, Icarus, 203, 681

Carvano, J. M., Barucci, M. A., Delbo, M., et al. 2008, A\&A 479, 241

Cellino, A., Gil-Hutton, R., Tedesco, E. F., Di Martino, M., \& Brunini, A. 1999, Icarus, 138, 129

Cellino, A., Belskaya, I. N., Bendjoya, Ph., et al. 2006, Icarus, 180, 565

Chapman C. R. 2004, Ann. Rev. Earth \& Planetary Sci., 32, 539

Chapman C. R., Morrison, D., \& Zellner, B. 1975, Icarus, 25, 104

DeMeo, F. E., Binzel, R. P., Slivan, S. M., \& Bus, S. J. 2009, Icarus, 202, 160

Dollfus, A., \& Geake, J. E. 1975, In Lunar Sci Conf. Proc., 6th, Houston, 3, 2749
Dollfus, A., Mandeville, J. C., \& Duseaux, M. 1979, Icarus, 37, 124 Dotto, E., Barucci, M. A., Fulchignoni, M., et al. 1992, A\&AS, 95, 195 Drummond, J. D., Conrad, A., Merline, W., \& Carry, B. 2009, DPS abstract, 41, 59.07

Feierberg, M. A., Larson, H. P., \& Chapman, C. R. 1982, ApJ, 257, 361 Feierberg, M. A., Witteborn, F. C., \& Lebofsky, L. A. 1983, Icarus, 56, 393 Fornasier, S., Belskaya, I., Shkuratov, Y. G., et al. 2006, A\&A, 455, 371 Fornasier, S., Migliorini, A., Dotto, E., \& Barucci, M. A. 2008, Icarus, 196, 119 Gaffey, M. J. 1976, JGR, 81, 905

Geake, J. E., \& Dollfus, A. 1986, MNRAS, 218, 75

Jonson, T. V., \& Fanale, F. P. 1973, JGR, 78, 8507

Gil-Hutton, R. 2007, A\&A, 464, 1127

Gil-Hutton, R., Mesa, V., Cellino, A., et al. 2008, A\&A, 482, 309

Kaasalainen, S., Piironen, J., Kaasalainen, M., et al. 2003, Icarus, 161, 24

Krugly, Yu. N., Belskaya, I. N., Shevchenko, V. G., et al. 2002, Icarus, 158, 294

Lagerkvist, C.-I., Erikson, A., Debehogne, H., et al. 1995, A\&AS, 113, 115

Landolt, A. U. 1983, AJ, 88, 439

Landolt, A. U. 1992, AJ, 104, 436

Lamy, P. L., Barucci, A., Fornasier, S., et al. 2008, In Asteroids, Comets, Meteors, LPI Contr., 1405

Lasker, B. M., Sturch, C. R., Lopez, C., et al. 1988, ApJS, 68, 1

Lazzarin, M., Marchi, S., Magrin, S., \& Barbieri, C. 2004, A\&A, 425, L25

Lazzarin, M., Marchi, S., Moroz, L. V., \& Magrin, S. 2009, A\&A, 498, 307

Lumme, K., \& Muinonen, K., 1993, Abstr. for the IAU Symp. 160, Asteroids, Comets, Meteors, 194

Lupishko, D. F., \& Belskaya, I. N. 1989, Icarus, 78, 395

Lupishko, D. F., \& Vasilyev, S. V. 2008, Asteroid Polarimetric Database V6.0, NASA PDS

Lupishko, D. F., Belskaya, I. N., \& Tupieva, F. A. 1983, Pis'ma Astron. Zh., 9, 691

Lupishko, D. F., Velichko, F. P., Belskaya, I. N., \& Shevchenko, V. G. 1987, Kinem. Fiz. Nebesn. Tel, 3, 36

Lupishko, D. F., Belskaya, I. N., Kvaratskheliia, O. I., Kiselev, N. N., \& Morozhenko, A. V. 1988, Astron. Vestnik, 22, 142

Magri, C., Ostro, S. J., Rosema, K. D., et al. 1999, Icarus, 140, 379

Masiero, J., \& Cellino, A. 2009, Icarus, 199, 333

Mishchenko, M. I., Rosenbush, V. K., \& Kiselev, N. N. 2006, Appl. Opt. IP, 45, 4459

Mottola, S., Gonano-Beurer, M., Green, S. F., et al. 1994, Planet. Space Sci., 42, 21

Mueller, M., Harris, A. W., Bus, S. J., et al. 2006, A\&A, 447, 1153

Muñoz, O., Volten, H., de Haan, J. F., Vassen, W., \& Hovenier, J. W. 2000, A\&A, 360,777

Nedelcu, D. A., Birlan, M., Vernazza, P., et al. 2007, A\&A, 470, 1157

Prokof'eva, V. V., Bochkov, V. V., \& Busarev, V. V. 2005, Solar System Res., 39, 410

Prokof'eva-Mikhailovskaya, V. V., Batrakov, Yu.V., Bochkov, V. V., Busarev, V. V., \& Karachkina, L. G. 2007, Solar System Res., 41, 307

Rivkin, A. S., Howell, E. S., Lebofsky, L. A., Clark, B. E., \& Britt, D. T. 2000, Icarus, 145, 351

Scott, E. R. D., \& Krot, A. N. 2005, ASP Conf. Ser., 341

Shepard, M. K., Clark, B. E., Nolan, M. C., et al. 2008, Icarus, 195, 184

Shepard, M. K., Clark, B. E., Oskert-Bell, M., et al. 2010, Icarus, in press

Shevchenko, V. G., \& Tedesco, E. F. 2006, Icarus, 184, 211

Shkuratov, Yu. G., Akimov, L. A., \& Tishkovets, V. P. 1984, SvA Lett., 10, 331

Shkuratov, Yu. G., Muinonen, K., Bowell, E., et al. 1994, EMP, 65, 201

Shkuratov, Yu. G., Ovcharenko, A., Zubko, E., et al. 2002, Icarus, 159, 396

Sunshine, J., Kolokolova, L., Connolly, H. C., et al. 2008, BAAS, 40, 509

Tedesco, E. F., Noah, P. V., Noah, M., \& Price, S. D. 2002, AJ, 123, 1056

Tholen, D. J. 1989, in Asteroids II, ed. R. P. Binzel, T. Gehrels, \& M. S. Matthews (Tucson: Univ. of Arizona Press), 1139

Torppa, J., Kaasalainen, M., Michalowski, T., et al. 2003, Icarus, 164, 346

Vernazza, P., Brunetto, R., Binzel, R. P., et al. 2009, Icarus, 202, 477

Williams, J. G. 1989, in Asteroids II (Univ. of Arizona Press), 1034

Zappala, V., di Martino, M., Knezevic, Z., \& Djurasevic, G. 1984, A\&A, 130, 208

Zappala, V., Bendjoya, Ph., Cellino, A., Farinella, P., \& Froeschle, C. 1995, Icarus, 116, 291

Zellner, B., \& Gradie, J. 1976, AJ, 81, 262

Zellner, B., Leake, M., Lebertre, T., Duseaux, M., \& Dollfus, A. 1977, Proc. Lunar Sci. Conf., 8th, 1, Houston, Tex., 1091

Zubko, E., Petrov, D., Shkuratov, Yu., \& Videen, G. 2005, Appl. Opt. IP, 44, 6479 